\title{
PREPARACIÓN Y CARACTERIZACIÓN DE LA ZEOLITA MCM-22 Y DE SU PRECURSOR LAMINAR
}

Sibele B. C. Pergher*

Departamento de Química, Universidade Regional Integrada do Alto Uruguai e das Missões, Campus Erechim, Av. Sete de Setembro, 1621, 99700-000 Erechim - RS

Avelino Corma y Vicente Fornés

Instituto de Tecnología Química, Universidad Politécnica de Valencia, Avd. de los Naranjos , s/n, 46022 Valencia - España

Recebido em 30/8/02; aceito em 4/4/03

\begin{abstract}
PREPARATION AND CHARACTERIZATION OF MCM-22 ZEOLITE AND ITS LAYERED PRECURSOR. The layered precursor of MCM-22 was prepared with different Si/Al ratios: 15, 25, 50, 100 and $\infty$. Upon heat treatment these precursors form MCM22 zeolite. Both layered precursor and MCM-22 zeolite were characterized by several techniques: Chemical Analysis by Atomic Absorption Spectroscopy (AAS), X-Ray Diffraction (XRD), Thermo-gravimetric Analysis (TGA), Pore Analysis by $\mathrm{N}_{2}$ and $\mathrm{Ar}$ adsorption, Scanning Electron Microscopy (SEM), Infrared Spectroscopy (IR) and Temperature Programmed Desorption of ammonium (TPD)
\end{abstract}

Keywords: MCM-22; layered precursor; zeolites.

\section{INTRODUCIÓN}

En la síntesis de zeolitas nuevas, hay un interés permanente en la obtención de tamices moleculares de poros grandes y extragrandes que puedan ser activas en reacciones catalíticas donde intervienen moléculas de gran tamaño (reactivos o productos). Estos materiales pueden tener un impacto positivo en procesos importantes como FCC ("Fluid Catalytic Cracking"), hidrocraqueo o y también en química fina. Por esta razón se busca preparar materiales zeolíticos bien definidos, de elevada área externa y que también presenten alguna característica de encerramiento de materiales microporosos.

Esta idea fue aplicada especialmente en materiales del tipo MWW $^{1,2}$, cuya estructura crece en láminas y posibilita la obtención de un precursor zeolítico en el cual las láminas no están aún condensadas $^{3}$. Llegado a este punto, se puede obtener la estructura MWW dejando condensar las láminas. Ésta estructura presenta dos sistemas independientes de poros: uno formado por cavidades de 12MR (18 x $7 \AA$ ) conectadas por aberturas de 10MR; y otro definido por un sistema de canales sinusoidales de $10 \mathrm{MR}^{1,2}$. Este tipo de estructura microporosa, aparte de ser inaccesible a grandes moléculas, posee un "handicap" asociado a la formación de coque en las cavidades conectadas por pequeñas ventanas. Con el objetivo de superar estas limitaciones, investigadores da Mobil ${ }^{4}$ pilarizaron el precursor laminar y obtuvieron la estructura MCM-36, en la cual las cavidades de 18 x 7 Å no están cerradas, sino separadas en dos mitades por pilares. A través de la deslaminación del precursor laminar se obtuvo un nuevo material más accesible y una nueva estructura fue aclamada ITQ-2 ${ }^{5-9}$, en la que las cavidades de 18 x 7 Å no están cerradas, pero totalmente accesibles. Este material cuenta con una enorme área superficial externa, y un gran acceso a las cavidades, manteniendo el sistema de poros de 10MR.

A través del mismo precursor laminar se puede obtener los materiales MCM-22, MCM-36 e ITQ-2, considerando esto, el objetivo de este trabajo fue estudiar la preparación y caracterización del precursor zeolítico laminar y del material MCM-22.

*e-mail: pergher@uri.com.br

\section{PARTE EXPERIMENTAL}

El precursor laminar zeolítico del material MCM-22 fue preparado utilizando hexametilenimina, sílice (Aerosil 200), aluminato de sodio $\left(56 \% \mathrm{Al}_{2} \mathrm{O}_{3}, 37 \% \mathrm{Na}_{2} \mathrm{O}\right)$, hidróxido de sodio y agua MiliQ $^{1,10,11}$.

Se prepararon precursores laminares con varias relaciones $\mathrm{Si}$ / $\mathrm{Al}: 15,25,50,100 \mathrm{e} \infty$. En la Tabla 1 se encuentran las composiciones de gel, temperaturas y tiempos empleados para cada precursor laminar. El procedimiento de preparación, independientemente de la relación $\mathrm{Si} / \mathrm{Al}$ (exceptuándose el caso de $\mathrm{Si} / \mathrm{Al}=\infty$ ), es el mismo. A continuación se ilustra, con un ejemplo, el método de preparación del precursor laminar de relación $\mathrm{Si} / \mathrm{Al}=50$ :

$0,234 \mathrm{~g}$ de aluminato de sodio y $0,816 \mathrm{~g}$ de $\mathrm{NaOH}$ fueron disueltos en $103,45 \mathrm{~g}$ de $\mathrm{H}_{2} \mathrm{O}$. A esta solución se adicionan $6,358 \mathrm{~g}$ de hexametilenimina y $7,689 \mathrm{~g}$ de $\mathrm{SiO}_{2}$ y la mezcla se somete a vigorosa agitación durante 30 minutos. Después de este periodo, el gel es introducido en autoclaves de acero inoxidable con una funda interna de teflón, de $60 \mathrm{ml}$ de capacidad, y se mantienen a $135{ }^{\circ} \mathrm{C}$ durante 11 días en rotación de $60 \mathrm{rpm}$. La composición del gel final fue de: $\mathrm{SiO}_{2} / \mathrm{Al}_{2} \mathrm{O}_{3}=100 ; \mathrm{OH} / \mathrm{SiO}_{2}=0,10 ; \mathrm{Na} / \mathrm{SiO}_{2}=0,18 ; \mathrm{R} / \mathrm{SiO}_{2}=0,50$ ( $\mathrm{R}=$ Hexametilenimina) e $\mathrm{H}_{2} \mathrm{O} / \mathrm{SiO}_{2}=44,9$.

Tabla 1. Composición del gel, temperaturas y tiempos utilizados para la preparación de precursores laminares de la MCM-22

\begin{tabular}{lcccc}
\hline $\mathrm{Si} / \mathrm{Al}$ & 15 & 25 & 50 & $100 *$ \\
\hline $\mathrm{SiO}_{2} / \mathrm{Al}_{2} \mathrm{O}_{3}$ & 30 & 50 & 100 & 200 \\
$\mathrm{OH} / \mathrm{SiO}_{2}$ & 0,11 & 0,10 & 0,10 & 0,11 \\
$\mathrm{Na} / \mathrm{SiO}_{2}$ & 0,18 & 0,14 & 0,18 & 0,18 \\
$\mathrm{R} / \mathrm{SiO}_{2}$ & 0,50 & 0,35 & 0,50 & 0,50 \\
$\mathrm{H}_{2} \mathrm{O} / \mathrm{SiO}_{2}$ & 45 & 45 & 45 & 45 \\
Temperatura $\left({ }^{\circ} \mathrm{C}\right)$ & 150 & 150 & 135 & 135 \\
Tiempo (días) & 7 & 9 & 11 & 8 \\
\hline
\end{tabular}

*: con siembra (MCM-22 de $\mathrm{Si} / \mathrm{Al}=15)$ y otro template 
Después de enfriados los autoclaves en agua, las muestras fueron centrifugadas a $12000 \mathrm{rpm}$, lavadas con $\mathrm{H}_{2} \mathrm{O}$ miliQ hasta que el $\mathrm{pH}$ final del agua de lavado fue de $\leq 9,0$ y se secaron a $60{ }^{\circ} \mathrm{C}$.

En la preparación del precursor laminar de relación $\mathrm{Si} / \mathrm{Al}=\infty$ (ITQ1), se utilizó hidróxido de n,n,n-trimetil-1-adamantamonio (TMAda ${ }^{+} \mathrm{OH}^{-}$), TMA-OH, $\mathrm{H}_{2} \mathrm{O}$ y sílice en autoclave a $150^{\circ} \mathrm{C}$ durante 17 días $^{12,13}$.

La calcinación del precursor da lugar a formación de la zeolita MCM-22. El programa de temperaturas utilizado fue seguinte: Calentamiento desde la temperatura ambiente hasta $150{ }^{\circ} \mathrm{C}$ durante $2,5 \mathrm{~h}$, mantiene $150^{\circ} \mathrm{C}$ por $2,5 \mathrm{~h}$, calentamiento hasta $350^{\circ} \mathrm{C}$ durante $1,5 \mathrm{~h}$, mantiene en $350^{\circ} \mathrm{C}$ por $3 \mathrm{~h}$, calentamiento hasta $580{ }^{\circ} \mathrm{C}$ durante $5 \mathrm{~h}$, manteiene en $580{ }^{\circ} \mathrm{C}$ por $3 \mathrm{~h}$.

\section{Caracterización}

\section{Análisis químico}

La composición química de los sólidos se determinó por Espectrofotometría de Absorción Atómica (AAB). Para los análisis se toman $50 \mathrm{mg}$ de muestra sólida, se calcina a $950{ }^{\circ} \mathrm{C}$ (calentamiento durante $6 \mathrm{~h}$, se mantiene a $950{ }^{\circ} \mathrm{C}$ durante $3 \mathrm{~h}$ y se enfría hasta $200{ }^{\circ} \mathrm{C}$ ), posteriormente, se disgregan con $1 \mathrm{~cm}^{3}$ de $\mathrm{HF}(48 \%)$ y $1 \mathrm{~cm}^{3}$ de $\mathrm{HNO}_{3}$ $(60 \%)$ a $40{ }^{\circ} \mathrm{C}$ en botes de propileno herméticamente cerrados, durante $24 \mathrm{~h}$, hasta obtener una disolución completa. Posteriormente las disoluciones son enrasadas con agua miliQ hasta un volumen de 50 $\mathrm{ml}$. El aparato empleado fue un VARIAN SPECTRA A-10plus.

\section{Difracción de rayos- $X^{14}$}

El equipo utilizado fue un difractómetro PHILIPS X'PERT PW3719 equipado con un polarizador de grafito, rendijas automáticas con superficie total de barrido constante de $14 \mathrm{~mm}$, portamuestras giratorio, cámara de temperatura Anton Parr modelo HTK16 y detector proporcional. Se utilizó la radiación $\mathrm{K} \alpha$ del Cobre $(\lambda=$ $1,54184)$, y una potencia de excitación de $2 \mathrm{~kW}$. La velocidad angular del goniómetro fue de $0,02 \%$, y el intervalo usual de barrido fue de $2 \theta=1$ a $40^{\circ}$.

\section{Análisis termogravimétrico y termodiferencial}

Los análisis térmicos se realizaron en un equipo NETZCH STA 409 EP, equipado con crisoles de platino sin tapa para la colocación de las muestras y un termopar de tipo $\mathrm{S}$ (Pt 10\% Rh-Pt). Los análisis se llevaron a cabo entre 20 y $800^{\circ} \mathrm{C}$, con un flujo de aire seco de 100 $\mathrm{ml} / \mathrm{min}$ y una velocidad de calentamiento de $10{ }^{\circ} \mathrm{C} / \mathrm{min}$, como referencia se utilizó caolín (calcinado a $1100{ }^{\circ} \mathrm{C}$ ). En todos los casos se emplearon 20 mg de muestra y la misma cantidad de caolín.

\section{Propiedades texturales por adsorción de nitrógeno}

Para la obtención de estos datos de adsorción, se utilizó un aparato MICROMERITCS ASAP 2000. La muestra es pastillada y tamizada, y se utiliza la fracción entre 0,59-0,84 mm. Para los análisis se utilizan $250 \mathrm{mg}$ y las muestras son pretratadas a $400{ }^{\circ} \mathrm{C}$ y a vacío durante aproximadamente $16 \mathrm{~h}$. Se realizan medidas de adsorción/ desorción.

\section{Espectroscopia infrarroja}

El aparato utilizado fue un espectrofotómetro NICOLET 710 FTIR. Antes de la obtención de los espectros, la muestra (pastilla auto consistente de $10 \mathrm{mg} / \mathrm{cm}^{2}$ ) fue tratada a $400{ }^{\circ} \mathrm{C}$ y a vacío, hasta la total eliminación del $\mathrm{H}_{2} \mathrm{O}$ y del material orgánico adsorbido sobre el sólido. La adsorción de piridina se realizó a presión de 5 torr, siendo el exceso retirado a vacío mediante calentamientos consecutivos a 150,250 y $350{ }^{\circ} \mathrm{C}$, cada uno seguido por enfriamiento y adquisición del espectro.

\section{Microscopia eletrónica de barrido}

El método de preparación de las muestras que se utilizó en este trabajo consiste en "espolvorear" la muestra sobre una pequeña sección de cinta adhesiva de doble cara adherida al portamuestras, de esta manera queda asegurado un fino recubrimiento. La metalización se realizó mediante la técnica de metalización al vacío, recubriendo las muestras con una fina capa de oro. Las fotografías fueron tomadas en un microscopio ISI-DS 130, equipado con un sistema de microanálisis por energía dispersiva KEVEX.

\section{RESULTADOS Y DISCUSIÓN}

\section{Precursor zeolitico laminar}

Los precursores laminares del material MCM-22 fueron preparados con diferentes relaciones $\mathrm{Si} / \mathrm{Al}: 15,25,50,100$ y $\infty$; y respectivamente denominados: PM22-15, PM22-25, PM22-50, PM22-100 y $\mathrm{PM} 22-\infty$.

Los resultados del análisis químico y las respectivas relaciones $\mathrm{Si} / \mathrm{Al}$ calculadas se encuentran en la Tabla 2.

Tabla 2. Resultados del análisis químico

\begin{tabular}{lcccc}
\hline Muestra & $\mathrm{Al}_{2} \mathrm{O}_{3}(\%)$ & $\mathrm{Na}_{2} \mathrm{O}(\%)$ & $\mathrm{SiO}_{2}(\%)$ & $\mathrm{Si} / \mathrm{Al}$ \\
\hline PM22-15 & 5,416 & 0,056 & 94,528 & 14,8 \\
PM22-25 & 3,205 & 0,057 & 96,738 & 25,6 \\
PM22-50 & 1,851 & 0,244 & 97,905 & 44,9 \\
PM22-100 & 1,033 & 0,782 & 98,185 & 80,8 \\
PM22- $\infty$ & 0,023 & 0,038 & 99,94 & 3750 \\
\hline
\end{tabular}

El porcentaje de $\mathrm{SiO}_{2}$ se calcula por diferencia, teniendo en cuenta que el material sólido solamente posee óxidos de $\mathrm{Al}, \mathrm{Na}$ y $\mathrm{Si}$, a través de la ecuación: $\% \mathrm{SiO}_{2}=100-\left(\% \mathrm{Al}_{2} \mathrm{O}_{3}+\% \mathrm{Na}_{2} \mathrm{O}\right)$. Se observa que las relaciones $\mathrm{Si} / \mathrm{Al}$ obtenidas están próximas de los valores del gel de síntesis. Estas muestras poseen una muy buena reproducibilidad.

Los difractogramas de rayos X del precursor de la MCM-22 descrito en la bibliografía ${ }^{15}$ y el obtenido en este trabajo PM22-15 son comparados en la Tabla 3, comprobándose que el material sintetizado PM22-15 corresponde al precursor de la MCM-22.

Los difractogramas de rayos X de los materiales PM22-50 y M2250 (obtenido por la calcinación a $580{ }^{\circ} \mathrm{C}$ del precursor PM22-50) son presentados en la Figura 1.

Comparando los dos difractogramas, se puede observar que la posición angular de algunas reflexiones tales como las localizadas a $2 \theta=3,3^{\circ}$ y $6,6^{\circ}$ (en el difractograma de la PM22-50) indexadas como (001) y (002) respectivamente, desaparecen durante la calcinación (M22-50). Otras bandas permanecen prácticamente inalteradas, tales como las localizadas a $2 \theta=7,21^{\circ}, 25,12^{\circ}$ y $26,11^{\circ}$, indexadas como $(h 00)$ y $(h k 0)$. Este comportamiento indica cambios estructurales esencialmente envolviendo el eje c (perpendicular a las láminas). Por otro lado se observa que el difractograma del material antes de calcinar (PM22-50) presenta bandas anchas, y que al calcinar la muestra aumenta la resolución de todas las bandas en general, principalmente en la zona de valores comprendida entre $20-25^{\circ}$.

Pequeños cambios en el espectro del difractograma de una zeolita al calcinar, tales como un estrechamiento de las reflexiones, se suelen atribuir a los cambios que la eliminación del agente orgánico director de estructura (template) pueden producir, entre ellos ligeras variaciones de los parámetros de celda unidad. Sin embargo, la determinación de parámetros de celda unidad en muestras MCM-22 antes y después de calcinar pone de manifiesto que existe una 
Tabla 3. Comparación de las bandas de rayos-X entre el precursor de la MCM-22 (Si/Al=15) ${ }^{16}$ y PM22-15

\begin{tabular}{ccccccc}
\hline \multicolumn{3}{c}{ Precursor de la MCM-22 } & & \multicolumn{3}{c}{ PM22-15 } \\
\cline { 5 - 7 } $2 \theta$ & d $(\AA)$ & I/Io & & $2 \theta$ & d $(\AA)$ & I/Io \\
\cline { 5 - 7 } 3,1 & 28,5 & W & & 3,19 & 27,67 & M \\
6,53 & 13,53 & M & & 6,49 & 13,60 & S \\
7,14 & 12,38 & VS & & 7,21 & 12,24 & VS \\
7,94 & 11,13 & M & & 7,9 & 11,18 & M \\
9,67 & 9,14 & W-M & & 9,62 & 9,18 & M \\
12,85 & 6,89 & W & & 12,87 & 6,87 & W \\
13,26 & 6,68 & W & & - & - \\
14,36 & 6,17 & W & & - & - \\
14,70 & 6,02 & W & & 14,67 & 6,03 & W \\
15,85 & 5,59 & W & & 15,71 & 5,63 & W \\
19,00 & 4,67 & W & - & - & - \\
19,85 & 4,47 & W-M & 19,78 & 4,48 & M \\
21,56 & 4,12 & W & 21,59 & 4,11 & W-M \\
21,94 & 4,05 & W-M & 21,89 & 4,05 & M \\
22,53 & 3,95 & W-M & 22,52 & 3,94 & M \\
23,59 & 3,77 & W & 23,35 & 3,8 & W-M \\
24,98 & 3,57 & W-M & 25,06 & 3,55 & W-M \\
25,98 & 3,43 & S & 25,99 & 3,42 & S-VS \\
26,56 & 3,36 & M & 26,55 & 3,35 & M \\
29,15 & 3,06 & W & 29,13 & 3,06 & W \\
31,58 & 2,83 & W & 31,63 & 2,82 & W \\
32,34 & 2,76 & W & 32,67 & 2,74 & W \\
\hline & & & & &
\end{tabular}

VS: 60 - 100; S: 40 - 60; M: 20 - 40; W: 0 -20

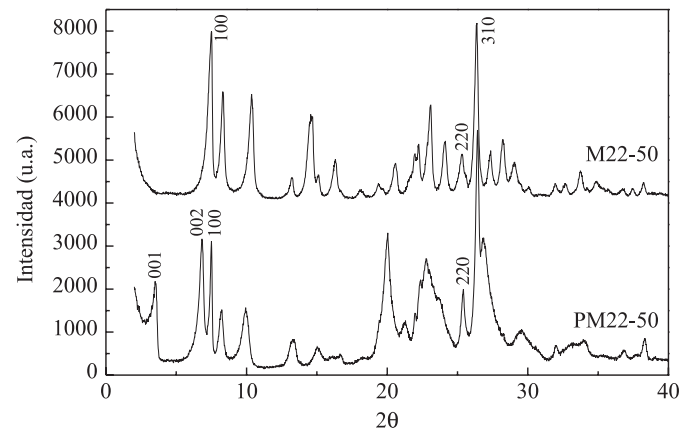

Figura 1. Difractogramas de rayos-X de las muestras PM22-50 y M22-50

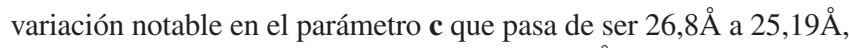
manteniendo a constante ${ }^{15}$. Este cambio de casi $2 \AA ̊$ no puede atribuirse a la relajación de la estructura de una zeolita tridimensional que se produce cuando se eliminan las moléculas de orgánico, por lo que se confirma que la zeolita MCM-22 antes de calcinar tiene una estructura laminar.

La Figura 2 ilustra una serie de difractogramas realizados en la cámara de temperatura sobre la PM22-15 a diferentes temperaturas. Los difractogramas fueron tomados a partir de $2 \theta=5,0^{\circ}$, por lo que sólo se observa la banda (002) de la fase laminar. La desaparición de esta banda indica la condensación de las láminas, formando así la M22-15. Se observa que su desaparición es gradual a partir de $150{ }^{\circ} \mathrm{C}$. A $200^{\circ} \mathrm{C}$ se observa aún esta banda y a partir de esta temperatura ya no es tan evidente su presencia.

La condensación de las láminas está representada esquemáticamente en la Figura 3. El precursor está constituido por láminas de $\sim 25 \AA$ de espesor, que incluyen una red de poros de $\sim 5 \AA$ de diámetro, separadas entre sí por moléculas de "template". La superficie externa de estas láminas está formada por grupos silanoles. Durante la

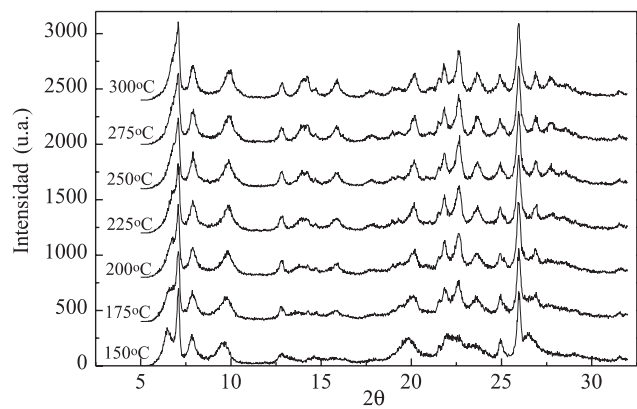

Figura 2. Difractograma de rayos-X de la muestra PM22-15 a diferentes temperaturas

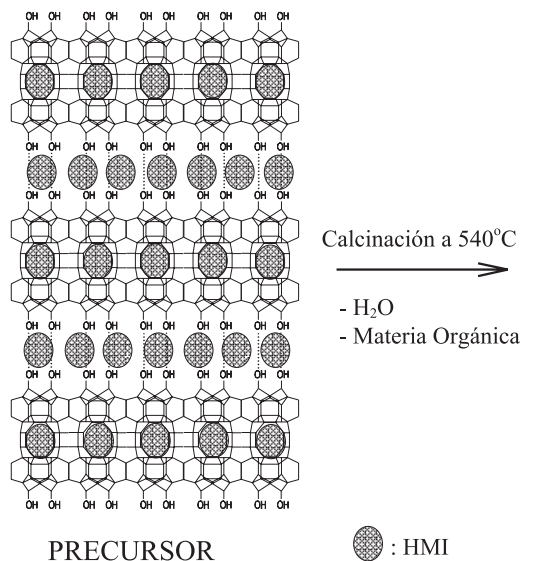

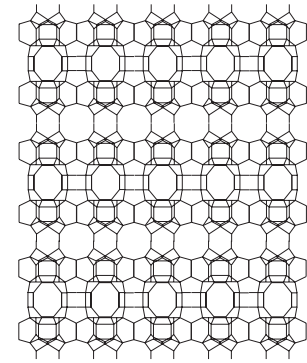

MCM-22
Figura 3. Esquema de condensación de las láminas del precursor formando así la MCM-22

calcinación esta materia orgánica es eliminada y tiene lugar una condensación de silanoles entre las láminas, con eliminación de agua, formando así una estructura tridimensional con dos sistemas de poros independientes: uno definido por canales de diez miembros sinusoidales y otro formado por supercavidades con diámetro interno de $7 \AA$.

La Figura 4 ilustra los difractogramas de los precursores con diferente relación Si/Al: PM22-15, -25, -50 y -100. Se observa que las intensidades de las bandas asignadas como (001) y (002) aumentan para materiales con mayor relación $\mathrm{Si} / \mathrm{Al}$, debido a que su cristalinidad aumenta con el aumento de la relación Si/Al.

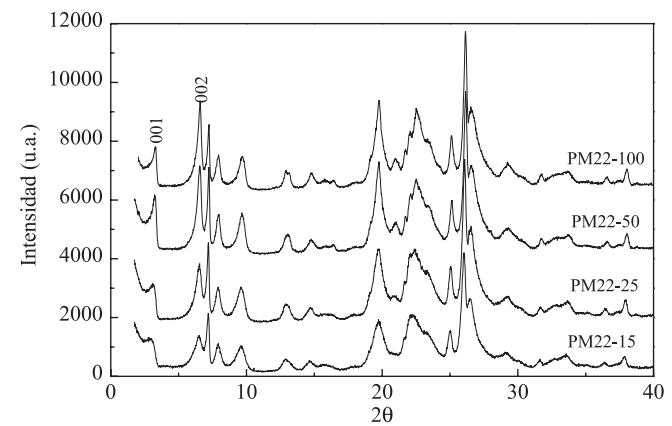

Figura 4. Difractogramas de rayos- $X$ de las muestras PM22-15, -25, - -50 y $-100$

Las curvas del análisis termodiferencial y termogravimétrico obtenidas para la muestra PM22-15 están representadas en la Figura 
5a. Se observa una pérdida inicial de $2,34 \%$ en peso hasta $\sim 200^{\circ} \mathrm{C}$ que se asocia a la pérdida de agua de hidratación. Entre 200 y $480{ }^{\circ} \mathrm{C}$ se observa una pérdida de $8,62 \%$ asociada a la pérdida de materia orgánica y agua procedente de la condensación de silanoles. Entre esas temperaturas se observan dos picos endotérmicos $\left(362{ }^{\circ} \mathrm{C}\right.$ y $417^{\circ} \mathrm{C}$ ) y un hombro entre 200 y $340^{\circ} \mathrm{C}$, indicando diferentes formas de asociación de la materia orgánica con el material. A partir de 480 hasta $740{ }^{\circ} \mathrm{C}$ se observa una pérdida de $7,37 \%$ y un pico endotérmico a $570{ }^{\circ} \mathrm{C}$ asociado a materia orgánica residual.
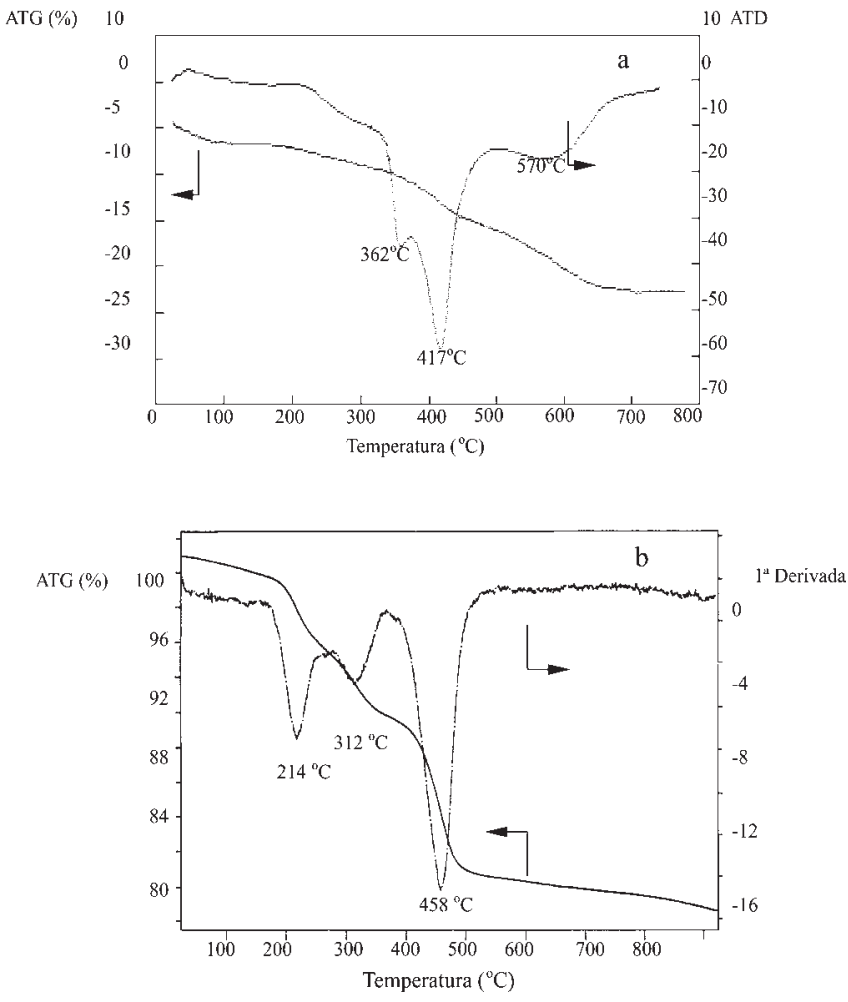

Figura 5. (a) Curvas de la análisis termogravimétrico (ATG) y termodiferencial (ATD) para la muestra PM22-15. (b) Curvas de la análisis termogravimétrico y termodiferencial para la muestra PM22-15

La materia orgánica (o template) utilizada para preparar el material PM22-15 fue la Hexametilenimina (HMI). La HMI puede estar localizada en los canales sinusoidales (intralaminar) o en la región interlaminar ${ }^{15} \mathrm{y}$ puede estar en la forma protonada o neutra.

Para obtener una mayor información sobre el proceso y asignar las pérdidas de peso, en las distintas temperaturas, a materia orgánica situada en los canales inter- o intralaminares del precursor, se realizó un análisis termodiferencial y termogravimétrico seguido de un análisis por espectrometría de masas para la PM22-15 y la PM22-15 intercambiada con $\mathrm{Na}^{+}$(que se supone que puede intercambiar completamente la materia orgánica protonada localizada en la región interlaminar). Se utilizó un ESA PERKIN-ELMER 7 Series Thermal Analysis Systems. La Figura 5b corresponde al espectro termogravimétrico para la muestra PM22-15 donde se observan principalmente tres picos endotérmicos: 214, 312 y $458{ }^{\circ} \mathrm{C}$. Hasta $\sim 190{ }^{\circ} \mathrm{C}$ se observa una pérdida inicial de $1,186 \%$ asociada al agua; al primer pico $\left(\sim 214^{\circ} \mathrm{C}\right)$ se asocia una pérdida de $4,561 \%$; al segundo, $3,41 \%$; y al último, 11,04\%.

Comparando las Figura 5a y Figura 5b se observan diferencias que son supuestamente debidas a los diferentes equipos y condiciones utilizados. El análisis termogravimétrico de la Figura 5a se obtuvo en flujo de aire mientras que el de la Figura 5b se realizó en flujo de He.
La conexión en línea del aparato termogravimétrico con un espectrómetro de masas permite convertir los resultados del análisis termodiferencial en tiempos de desorción en lugar de temperatura, como se ilustra en la Figura 6, obteniéndose el espectro de masas para cada pico de desorción.

En la Figura 6 se observan 4 picos que se denominaran I, II, III y IV. Los respectivos espectros de masas se encuentran representados en la Figura 7.

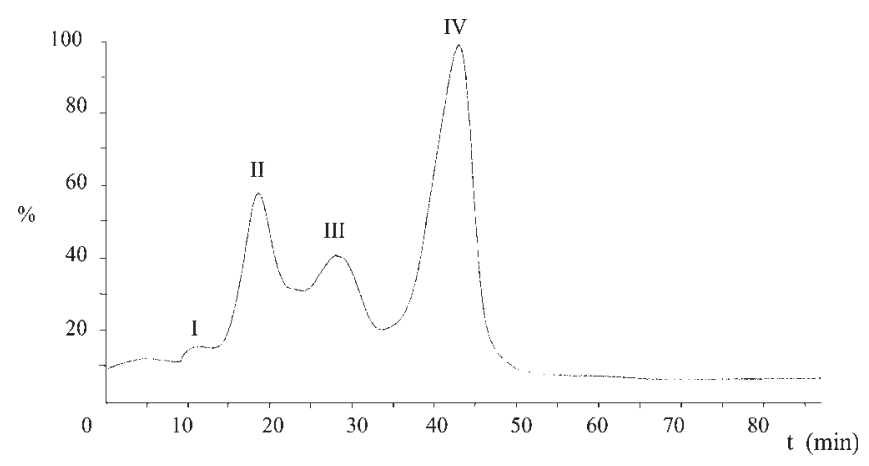

Figura 6. Curvas de análisis termodiferencial relativa a tiempos de desorción

Se identifica el pico numero I como $\mathrm{H}_{2} \mathrm{O}$ (masa 18), lo que confirma que la primera pérdida de peso $\sim 1,2 \%$ que ocurre hasta $\sim 190^{\circ} \mathrm{C}$ es de $\mathrm{H}_{2} \mathrm{O}$ de hidratación. Los picos denominados como II y III presentan un espectro de masas similar al correspondiente a la HMI (masas 30, 43, 56, 70 y 99), confirmando que la segunda y tercera pérdidas de peso deben ser asignadas a la eliminación de la HMI (con agua) aunque no se puede diferenciar si están en forma protonada o neutra. La Figura 8 representa una propuesta de fragmentación para la HMI donde se explican las bandas que aparecen en el espectro de masas. El último pico IV, presenta un particular espectro de masas de difícil interpretación, aunque probablemente sea debido a que la HMI interacciona entre sí y se descompone térmicamente dando lugar a una mezcla de compuestos.

Los picos II y III son asignados a la HMI interlaminar, pues se desorben a temperaturas relativamente bajas. Además, al mismo tiempo tiene lugar una pérdida de agua correspondiente a la condensación de silanoles que están en la región interlaminar. El pico IV por estar en una región de temperaturas altas $\left(400-500{ }^{\circ} \mathrm{C}\right)$ es asignado a la HMI intralaminar, donde la molécula estaría atrapada en el canal y sólo podría ser desorbida por descomposición. Por ese motivo no se observa un espectro de masas claro como en los casos anteriores.

En la muestra PM22-15 intercambiada con $\mathrm{Na}^{+}$se observa una disminución de intensidad en el pico numero II de 4,56\% frente a $1,883 \%$ (Figura 9), siendo el resto de los picos muy similares a la muestra original. Según esos resultados y considerando las temperaturas a las que se producen las pérdidas de peso, se propone que el pico II corresponda a la HMI protonada que está muy accesible en la región interlaminar, donde gran parte de la misma es susceptible de ser intercambiada por $\mathrm{Na}^{+}$. El pico III se asignaría a la HMI neutra situada en la región interlaminar. Por otro lado, teniendo en cuenta que entre 200 y $300{ }^{\circ} \mathrm{C}$ se produce el colapso de las láminas con formación de las supercavidades, la curva obtenida podría explicarse suponiendo un colapso parcial en este rango de temperaturas: el pico II correspondería a HMI interlaminar en el precursor mientras que el pico III se asignaría a HMI en el interior de las supercavidades formadas al colapsar la estructura. En una segunda hipótesis podemos considerar que el precursor está formado inicialmente por una mezcla de compuesto laminar y MCM-22 ya colapsada ${ }^{16}$ y la asignación de los picos sería la misma. 

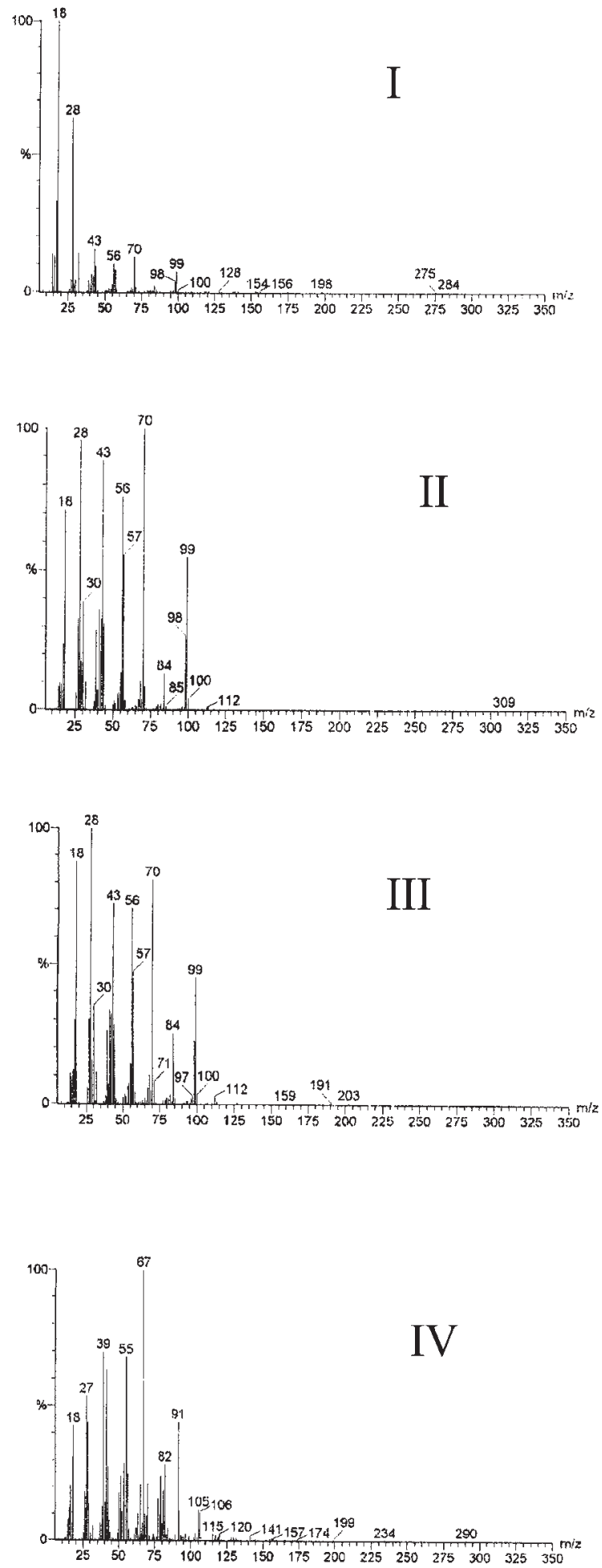

Figura 7. Espectros de masas obtenidos para los picos asignados en la Figura 6

Finalmente, el cuarto pico estaría compuesto por ambas HMI, protonada y no protonada, atrapadas dentro del canal de 10MR, las cuales sólo consiguen difundir al exterior previa fragmentación.

\section{MCM-22}

Los precursores laminares preparados tal y como se ha descrito y caracterizados anteriormente fueron calcinados a $580{ }^{\circ} \mathrm{C}$ formando

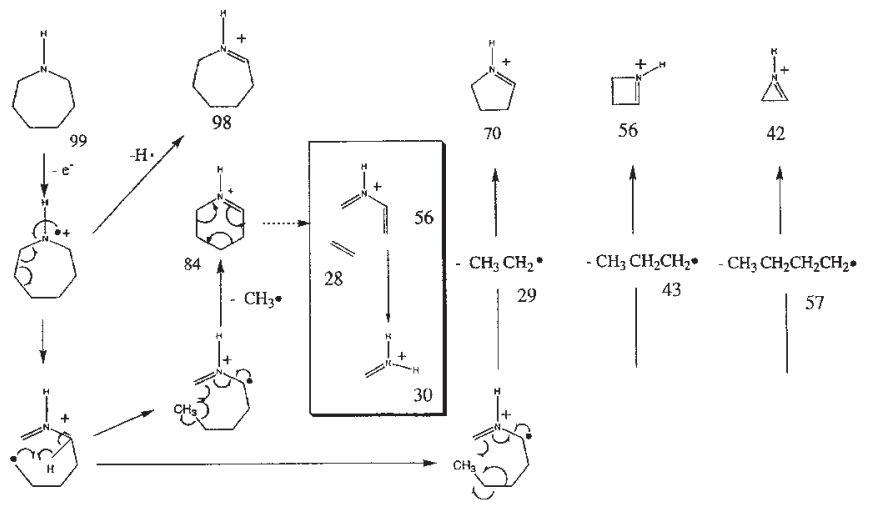

Figura 8. Esquema de fragmentación propuesta para la HMI

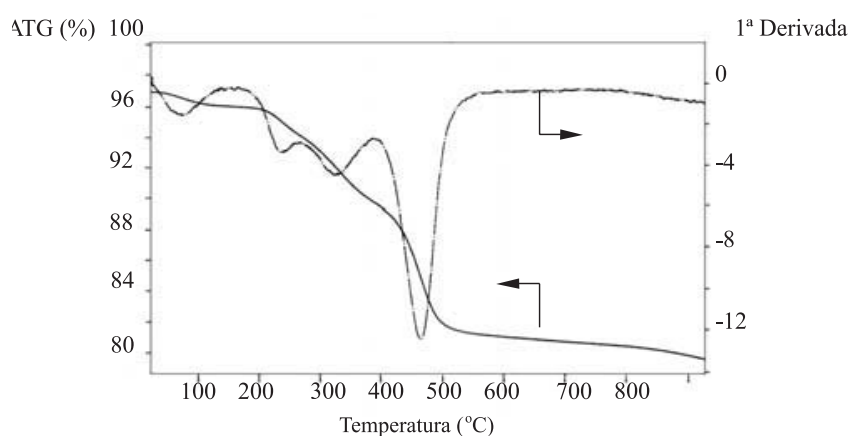

Figura 9. Curvas del análisis termogravimétrico y termo-diferencial para la muestra PM22-15 intercambiada con $\mathrm{Na}^{+}$

así el material MCM-22.

Los materiales preparados con diferentes relaciones $\mathrm{Si} / \mathrm{Al}$ : 15, $25,50,100$ y $\infty$; fueron respectivamente denominados: M22-15, M2225, M22-50, M22-100 у M22- $\infty$.

Los análisis químicos de las muestras M22 dieron los mismos resultados presentados para los precursores PM22 respectivos.

En la Figura 10 se presentan los difractogramas de las muestras M22-15 y M22-50, observándose que con el aumento de la relación $\mathrm{Si} / \mathrm{Al}$ aumenta la definición de las bandas, indicando el aumento de la cristalinidad.

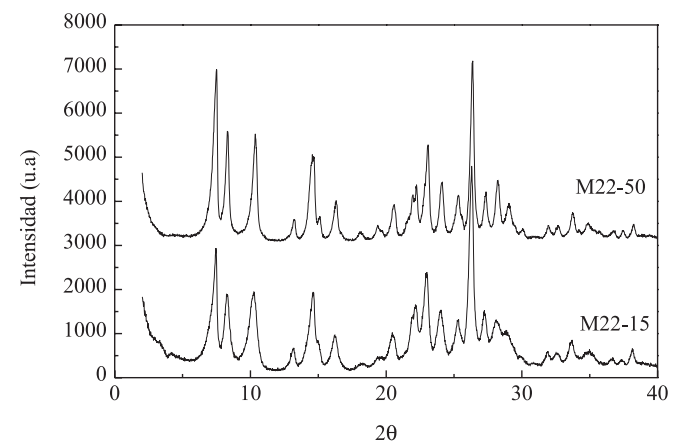

Figura 10. Difractogramas de rayos-X de las muestras M22-15 y M22-50

Una comparación entre la posición e intensidad de los picos de difracción correspondientes a la MCM-22 según la patente ${ }^{1}$ y la obtenida en este trabajo se muestra en la Tabla 4. Se observa que el material sintetizado corresponde con el de la patente.

La isoterma de adsorción de $\mathrm{N}_{2}$ para la muestra M22-50 es representada en la Figura 11. Se observa que para p/po bajos tiene lugar una gran adsorción de $\mathrm{N}_{2}$, comportamiento típico de materiales 
Tabla 4. Comparación de bandas de difracción de rayos-X entre la MCM-22 (Si/Al=15) (Patente) y M22-15

\begin{tabular}{|c|c|c|c|c|c|}
\hline & MCM-22 & & & M22-15 & \\
\hline $2 \theta$ & $\mathrm{d}(\AA)$ & I/Io & $2 \theta$ & $\mathrm{d}(\AA)$ & I/Io \\
\hline 2,80 & 31,55 & M & - & - & - \\
\hline 4,02 & 21,98 & W & - & - & - \\
\hline 7,10 & 12,45 & VS & 7,14 & 12,37 & VS \\
\hline 7,95 & 11,12 & $\mathrm{~S}$ & 7,93 & 11,14 & $\mathrm{~S}$ \\
\hline 10,00 & 8,85 & S & 10,025 & 8,81 & $\mathrm{~S}$ \\
\hline 12,90 & 6,86 & W & 12,860 & 6,878 & W \\
\hline 14,34 & 6,18 & $S$ & 14,36 & 6,16 & $\mathrm{~S}$ \\
\hline 14,72 & 6,02 & W & 14,735 & 6,007 & W \\
\hline 15,90 & 5,57 & W-M & 15,96 & 5,54 & W-M \\
\hline 17,81 & 4,98 & W & 17,77 & 4,98 & W \\
\hline 19,08 & 4,65 & W & 19,01 & 4,66 & W \\
\hline 20,20 & 4,40 & W-M & 20,29 & 4,37 & W-M \\
\hline 20,91 & 4,25 & W & - & - & - \\
\hline 21,59 & 4,12 & W-M & 21,575 & 4,11 & W-M \\
\hline 21,92 & 4,06 & W & 21,88 & 4,058 & W \\
\hline 22,67 & 3,92 & M & 22,76 & 3,90 & M \\
\hline 23,70 & 3,75 & W & 23,745 & 3,74 & W \\
\hline 25,01 & 3,56 & W & 24,97 & 3,56 & W \\
\hline 26,00 & 3,43 & VS & 26,060 & 3,41 & VS \\
\hline 26,96 & 3,31 & W & 26,98 & 3,30 & $\mathrm{~W}$ \\
\hline 27,75 & 3,21 & W & 27,88 & 3,19 & W \\
\hline 28,52 & 3,13 & W & 28,705 & 3,10 & W \\
\hline 29,01 & 3,08 & W & - & - & - \\
\hline 29,71 & 3,01 & W & 29,69 & 3,006 & W \\
\hline 31,61 & 2,830 & W & 31,56 & 2,83 & W \\
\hline 32,21 & 2,779 & W & 32,33 & 2,767 & W \\
\hline 33,35 & 2,687 & W & 33,485 & 2,674 & W \\
\hline 34,61 & 2,592 & W & 34,485 & 2,5987 & W \\
\hline
\end{tabular}

VS: 60 - 100; S: 40 - 60; M: 20 - 40; W: 0 - 20

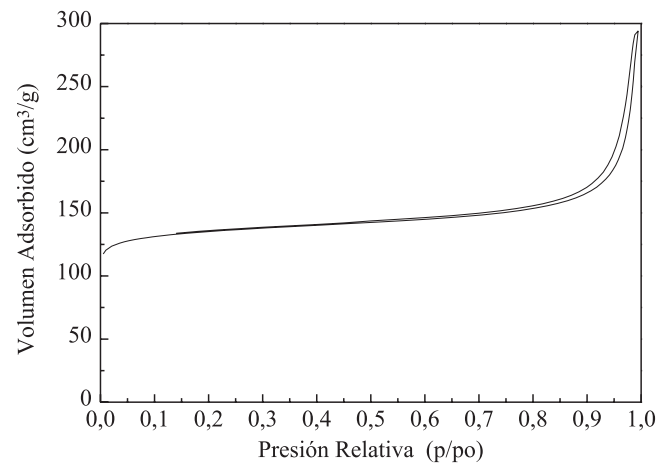

Figura 11. Isoterma de adsorción de $N_{2}$ para la muestra M22-50 microporosos. Para p/po más elevados ya se observa una pequeña histéresis indicando que el material también contiene mesoporos.

A partir de la isoterma, se calculan la superficie específica y el volumen de poro. La Tabla 5 muestra estos resultados para las diferentes muestras. La superficie específica $\mathrm{BET}\left(\mathrm{S}_{\mathrm{BET}}\right)$ es el área "total" del material calculada por el método BET, mientras que el área representativa de los microporos $\left(\mathrm{S}_{\text {micro }}\right)$ se calcula por el método $t$ plot. La diferencia entre ambas áreas corresponde a la contribución de la superficie "externa" $\left(\mathrm{S}_{\text {ext }}\right)$ que incluirá meso y macroporos. El volumen total $\left(\mathrm{V}_{\text {total }}\right)$ es el volumen adsorbido hasta p/po 0,99, mientras que el volumen de microporo $\left(\mathrm{V}_{\text {micro }}\right)$ se calcula por el método t-plot. El volumen representativo de los mesoporos (entre $17 \mathrm{y}$ $300 \AA ̊$ ) se calcula por el método BJH.

Se observa una superficie específica BET promedio de unos $450 \mathrm{~m}^{2} / \mathrm{g}$, de los cuales un mínimo de $310 \mathrm{~m}^{2} / \mathrm{g}$ corresponden a la microporosidad. Se observa que con el aumento de la relación $\mathrm{Si} / \mathrm{Al}$ tiene lugar un aumento de la microporosidad y disminución de la $\mathrm{S}_{\text {ext }}$, debido al aumento de la cristalinidad y del tamaño de los cristales. El material M22- $\infty$ fue sintetizado por otro método razón por lo cual su cristalinidad es menor.

En la Figura 12 se representa la distribución de tamaño de poro según la ecuación de Havarth-Kawazoe, obtenida a partir del análisis de adsorción de argón. Se observa una distribución de tamaño de poro centrada entre 5 - $7 \AA$. El poro de $\sim 5 \AA$ es atribuido al sistema de canales sinusoidales de $10 \mathrm{MR}$ y a las supercavidades con entrada de $10 \mathrm{MR}$.

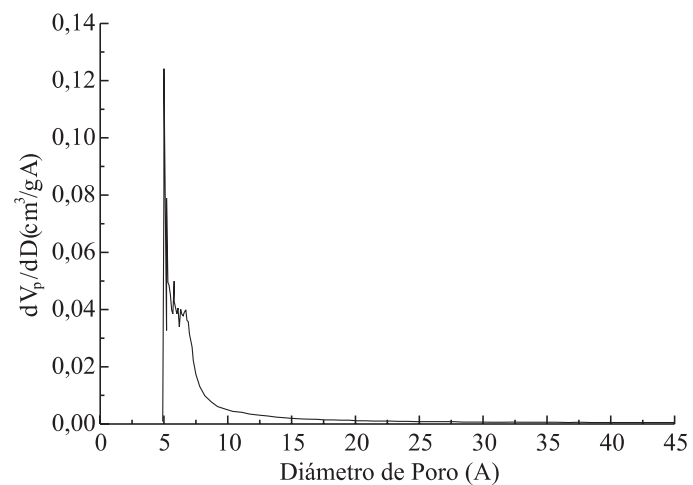

Figura 12. Distribución de tamaño de poro según la ecuación de HavarthKawazoe para el material M22

En las fotografías de microscopía electrónica de las muestras M22-15 y M22-50 (Figura 13) se puede observar que la morfología de los cristales es semejante a monedas, lo que indicaría un cierto hábito laminar: ejes a y b mucho mayores que c. El tamaño promedio de los cristales para la muestra M22-15 puede ser estimado alrededor de 0,3 - 0,5 um de diámetro, siendo el grosor de estas láminas mucho menor variando entre $0,03-0,05 \mu \mathrm{m}$. La muestra M22-50 presenta cristales mayores alrededor de $0,8-1 \mu \mathrm{m}$, de acuerdo con la mayor

Tabla 5. Superficie específica y volumen de poro calculados para las muestras con diferentes relaciones $\mathrm{Si} / \mathrm{Al}$

\begin{tabular}{lcccccc}
\hline Muestra & $\mathrm{S}_{\text {BET }}\left(\mathrm{m}^{2} / \mathrm{g}\right)$ & $\mathrm{S}_{\text {micro }}\left(\mathrm{m}^{2} / \mathrm{g}\right)$ & $\mathrm{S}_{\text {ext }}\left(\mathrm{m}^{2} / \mathrm{g}\right)$ & $\mathrm{V}_{\text {total }}\left(\mathrm{cm}^{3} / \mathrm{g}\right)$ & $\mathrm{V}_{\text {micro }}\left(\mathrm{cm}^{3} / \mathrm{g}\right)$ & $\mathrm{V}_{\text {BJH }}\left(\mathrm{cm}^{3} / \mathrm{g}\right)$ \\
\hline M22-15 & 453 & 312 & 141 & 0,589905 & 0,155190 & 0,311989 \\
M22-25 & 448 & 339 & 109 & 0,528903 & 0,169119 & 0,208137 \\
M22-50 & 452 & 355 & 97 & 0,523984 & 0,177122 & 0,169253 \\
M22-100 & 455 & 378 & 77 & 0,551616 & 0,189095 & 0,110720 \\
M22- $\infty$ & 303 & 195 & 108 & 0,628357 & 0,097196 & 0,302362 \\
\hline
\end{tabular}



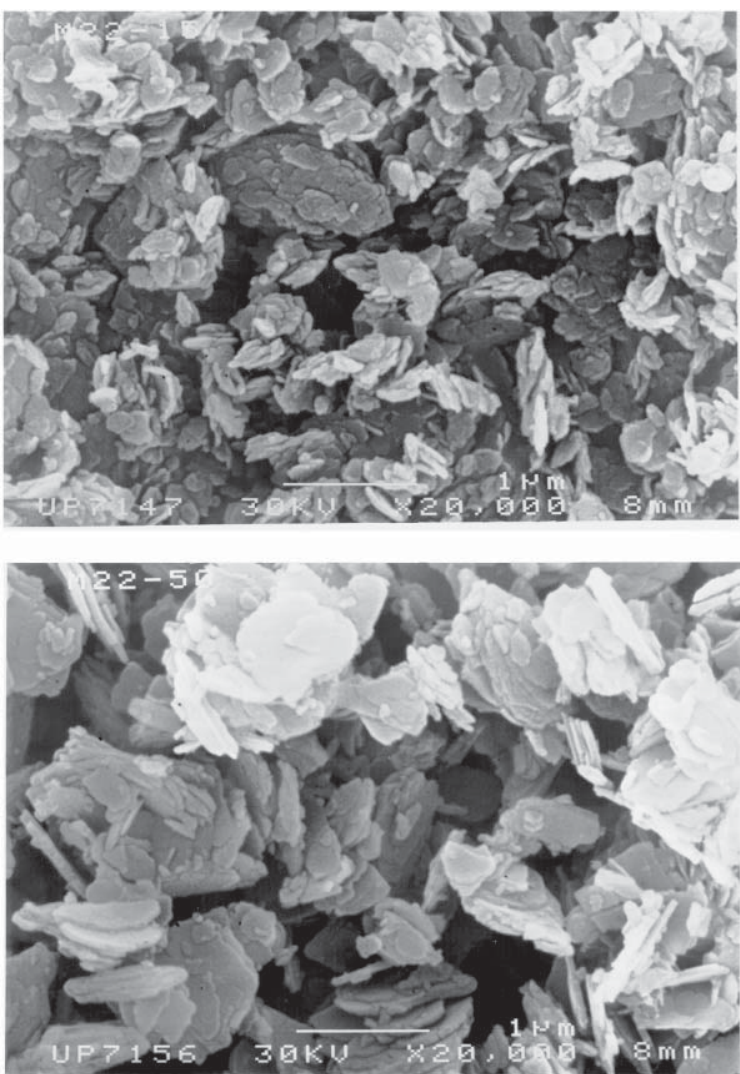

Figura 13. Fotografías obtenidas por microscopía electrónica de barrido de las muestras M22-15 (arriba) y M22-50 (abajo)

cristalinidad que presenta este material al comparar con la M22-15.

La acidez de los materiales fue evaluada por dos técnicas: espectroscopia infrarroja de muestras con piridina adsorbida y desorción de amoníaco a temperatura programada.

El espectro de infrarrojo en la zona de hidroxilos obtenido para las muestras M22-15 y M22-50 está representado en la Figura 14. Se observa que con la adsorción de piridina tiene lugar la desaparición de la banda a $\sim 3620 \mathrm{~cm}^{-1}$, correspondiente a los grupos $\mathrm{OH}$ asociados a aluminio intrarreticular, indicando que todos los centros ácidos de la zeolita son accesibles. Sin embargo, las bandas asociadas a grupos silanoles $\left(3747 \mathrm{~cm}^{-1}\right)$ varían poco de intensidad y ponen de manifiesto que se trata de centros con un carácter ácido muy débil.

En las dos muestras aparece una banda a $\sim 3747 \mathrm{~cm}^{-1}$ de silanoles externos y un hombro y/o banda a 3700 y $3500 \mathrm{~cm}^{-1}$ que corresponde a silanoles internos, siendo estas últimas más patentes al aumentar la relación Si/Al. Este tipo de bandas asociadas a silanoles internos pueden verse afectadas por la localización de éstos en la estructura. Así, teniendo en cuenta que en esta zeolita existen supercavidades delimitadas por anillos de $12 \mathrm{MR}$ y canales de $10 \mathrm{MR}$, y considerando que este tipo de bandas aparecen en la zeolita $\mathrm{Y} \mathrm{a} \sim 3700 \mathrm{~cm}^{-1} \mathrm{y}$ en la ZSM-5 centradas en $\sim 3500 \mathrm{~cm}^{-1}{ }^{16-18}$, la banda que aparece a mayor frecuencia de vibración podría corresponder a los silanoles en defectos producidos en las supercavidades, mientras que los que aparecen $\mathrm{a} \sim 3500 \mathrm{~cm}^{-1}$ se asignarían a defectos en los canales de 10 MR similares a los de ZSM-5. Al disminuir el contenido en aluminio de las muestras la cantidad de silanoles externos decrece, aumentando la de silanoles internos.

La Figura 15 presenta el espectro infrarrojo en la zona de 1750 a $1350 \mathrm{~cm}^{-1}$ para la muestra M22-15 después de la adsorción de piridina y sucesivos calentamientos a 250,350 y $400{ }^{\circ} \mathrm{C}$. Se observa acidez Lewis $\left(1622\right.$ y $\left.1454 \mathrm{~cm}^{-1}\right)$ y acidez Brönsted $\left(1548\right.$ y $\left.1639 \mathrm{~cm}^{-1}\right)$.
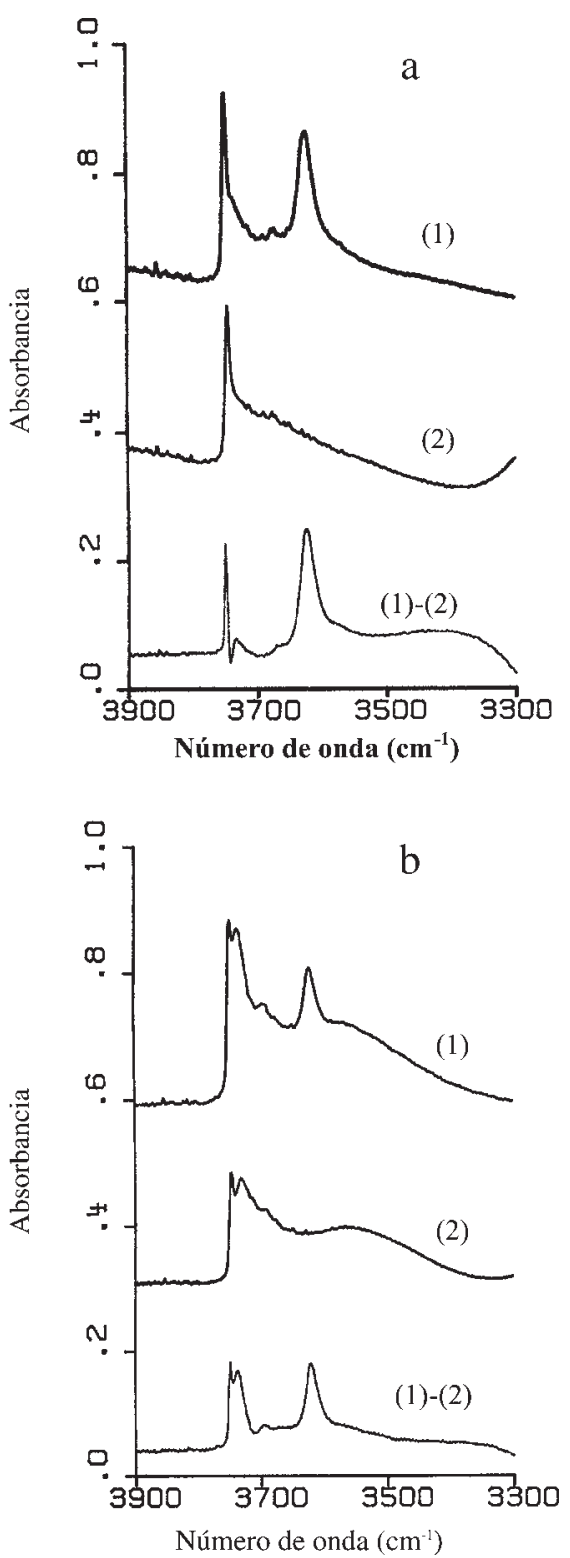

Figura 14. Espectro de infrarrojo obtenido en la zona de hidroxilos para las muestras M22-15 (a) y M22-50 (b), donde: (1) espectro registrado después de tratar la muestra a $400{ }^{\circ} \mathrm{C}$ y a vacío y (2) después de adsorber piridina y desorberla a $250{ }^{\circ} \mathrm{C}$

La Tabla 6 presenta las cantidades de piridina adsorbida en los centros Lewis y Brönsted a diferentes temperaturas para las muestras M22-15 y M22-50. Se observa claramente que la muestra M22-15 posee más acidez Brönsted y Lewis, lo que era de esperar por su mayor cantidad de aluminio.

Por otro lado, la cantidad de amoníaco adsorbido está relacionada con el número de centros ácidos del material (Lewis y Brönsted). El amoníaco posee un diámetro cinético (Lennard - Jones) de $\sim 2,6 \AA$, por eso no tiene dificultad en difundir a través los poros formados por los anillos de 10 miembros.

Las curvas de desorción temoprogramada de $\mathrm{NH}_{3}$ para las muestras M22-15 y M22-50 están representadas en la Figura 16. La cantidad de aluminio en el material está directamente relacionada con la acidez, y por lo tanto con la cantidad de $\mathrm{NH}_{3}$ desorbida. $\mathrm{La}$ relación entre las cantidades desorbidas es 29,85/10,55 $=2,83$, indicando que la muestra M22-15 posee 2,83 veces más centros ácidos totales que la M22-50. 


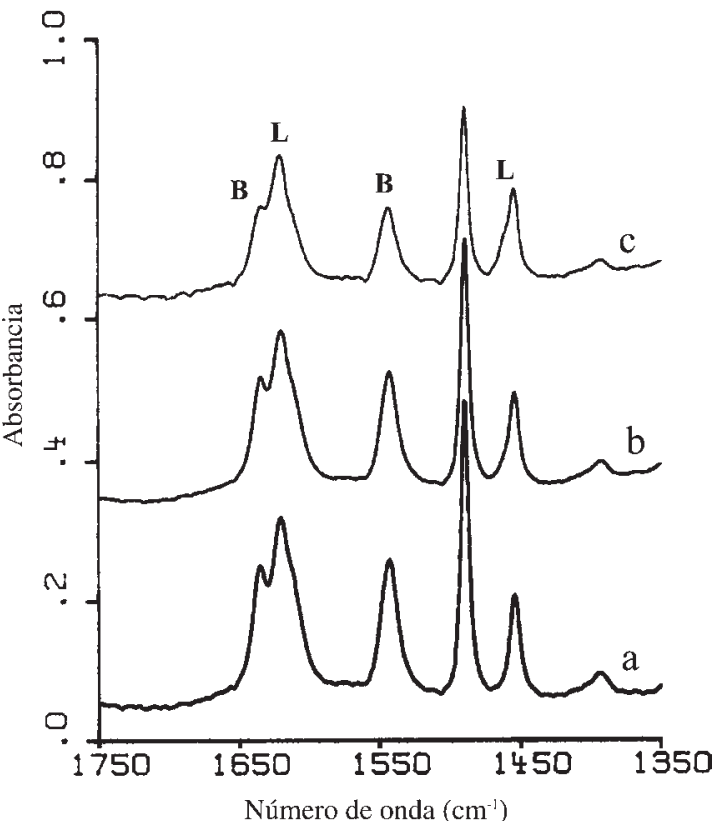

Figura 15. Espectro de infrarrojo de la muestra M22-15 después de adsorber piridina y desorberla a (a) $250^{\circ} \mathrm{C}$, (b) $350{ }^{\circ} \mathrm{C}$ y (c) $400{ }^{\circ} \mathrm{C}$

Tabla 6. Cantidades de piridina adsorbida ( $\mathrm{mmol} / \mathrm{g}$ muestra) en los centros ácidos Lewis y Brönsted a diferentes temperaturas para las muestras M22-15 y M22-50

\begin{tabular}{lcccccc}
\hline & \multicolumn{2}{c}{$250{ }^{\circ} \mathrm{C}$} & \multicolumn{2}{c}{$350{ }^{\circ} \mathrm{C}$} & \multicolumn{2}{c}{$400{ }^{\circ} \mathrm{C}$} \\
& Lewis & Brönsted & Lewis & Brönsted & Lewis & Brönsted \\
\hline M22-15 & 22,6 & 55,6 & 20,2 & 48,3 & 20,2 & 32,6 \\
M22-50 & 15,6 & 25,4 & 15,0 & 15,7 & 9,2 & 7,5 \\
\hline
\end{tabular}

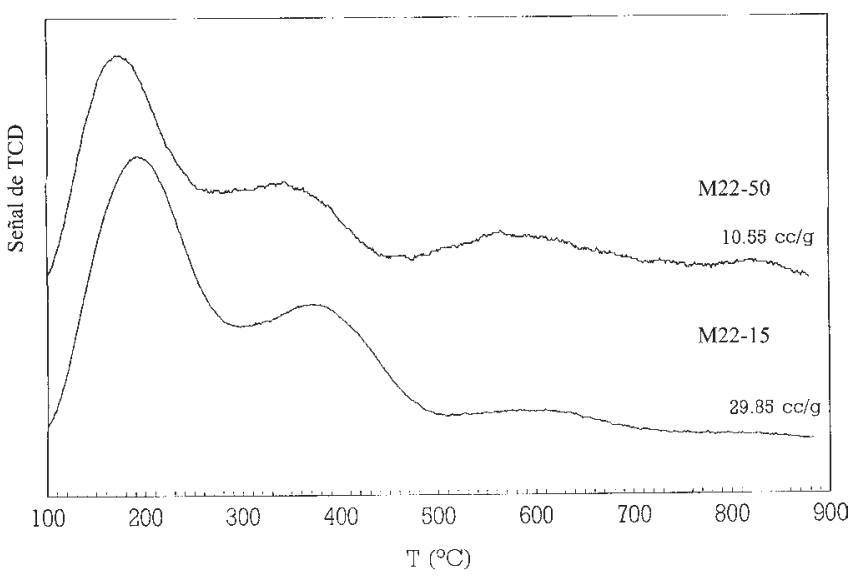

Figura 16. Curvas de desorción de amoníaco a temperatura programada para las muestras M22-15 y M22-50

\section{CONCLUSIÓN}

El precursor laminar de la MCM-22 es identificado por su difractograma de rayos $\mathrm{X}$ característico, donde se observan las bandas asignadas como 001 y 002 correspondientes a un compuesto laminar. Además, sus bandas de difracción son en general más anchas que las del material calcinado (MCM-22). El precursor posee aproximadamente $20 \%$ de materia orgánica (HMI) que está distribuida entre la región interlaminar y los canales de 10MR sinusoidales. Se puede decir que la HMI que está en los canales de 10MR está atrapada y sólo puede ser eliminada por descomposición de la misma por tratamientos a temperaturas elevadas. La HMI interlaminar puede ser intercambiada al menos parcialmente por otras moléculas y/o cationes.

El material M22 se obtiene por la calcinación de su precursor PM22 y es identificado por su difractograma de rayos X característico. Se observa un aumento de la cristalinidad con el aumento de la relación $\mathrm{Si} / \mathrm{Al}$, en consecuencia, también aumenta el tamaño de los cristales. El material posee un área promedio de $450 \mathrm{~m}^{2} / \mathrm{g}$, de los cuales un mínimo de $310 \mathrm{~m}^{2} / \mathrm{g}$ son debidos a microporosidad, con tamaños de poros entre 5-7 $\AA$. Los materiales presentan acidez Brönsted y Lewis, siendo mayor la cantidad de centros ácidos para las muestras con menor relación $\mathrm{Si} / \mathrm{Al}$.

\section{REFERENCIAS}

1. Rubin, M. K.; Chu, P.; US pat. 49543251990.

2. Leonowicz, M. E.; Lawton, J. A.; Lawton; S. L.; Rubin; M. K.; Science 1994, 264, 1910.

3. Milini, R.; Perego, G.; Parker Jr., W. O.; Bellusi, G.; Carluccio, L.; Microporous Mesoporous Mater. 1995, 4, 221.

4. Kresge, C. T.; Roth, W. L.; Simons, K. G.; Vartuli, J. C.; Word pat. WO92/ 119341992.

5. Pergher, S. B. C.; Tese de Doutorado, Universidad Politécnica de Valencia, Espanha, 1997.

6. Corma, A.; Fornés, V.; Guil, J. M.; Martinez-Triguero, J.; Pergher, S. B. C.; Maessen, Th. L. M.; Buglass, J. G.; Microporous Mesoporous Mater. 2000, 38, 301 .

7. Corma, A.; Fornes, V.; Martinez- Triguero, J.; Pergher, S. B. C.; J. Catal. 1999, 186, 57.

8. Corma, A.; Fornes, V.; Pergher, S. B. C.; Maessen, Th. L. M.; Buglass, J. G.; Nature 1998, 396, 353.

9. Pergher, S. B. C.; Corma, A.; Fornes, V.; XVIII Simpósio Iberoamericano de Catálisis, Porlamar - Isla Margarita, Venezuela, 2002.

10. Corma, A.; Corell,C.; Perez-Pariente, J.; Zeolites 1995, 15, 2.

11. Corma, A.; Corell, C.; Fornes, V.; Kolodziejski, W.; Perez-Pariente, J.; Zeolites 1995, 15, 576.

12. Camblor, M. A.; Corell, C.; Corma, A.; Díaz-Cabañas, M. J.; Nicolopoulos, S.; González-Calbet, J. M.; Vallet-Regí, M.; Chem. Mater. 1996, 8, 2415.

13. Camblor, M. A.; Díaz-Cabañas, M. J.; Corell, C.; Corma, A.; Pat. Española P9501553 1995.

14. Gallego, M. R. Em La Difracción de los Rayos X; Alhambra: Madrid, 1982.

15. Lawton, S. L.; Fung, A. S.; Kennedy, G. J.; Alemany, L. B.; Chang, C. D.; Hatzikos, G. H.; Lissy, D. N.; Rubin, M. K.; Timken, H. K. C.; Steuernagel, S.; Woessner, D. E.; J. Phys. Chem. 1996, 100, 3788.

16. Lohse, U.; Löffler, E.; Hunger, M.; Stöcker, J.; Patzelová, V.; Zeolites 1987, 7,11

17. Jacobs, P. A.; Uytterhoeven, J. B.; J. Catal. 1972, 22 , 193.

18. Woolery, G. L.; Alemany, L. B.; Dessau, R. M.; Chester, A. W.; Zeolites 1986, 6,14 . 